\title{
Monitoramento e Avaliação da Borracha Natural Crua Utilizando a Técnica de Análise Térmica Dinâmico-Mecânica
}

\author{
Rogério M. B. Moreno, Luiz Henrique C. Mattoso \\ Embrapa Instrumentação Agropecuária, São Carlos/SP \\ Aldo Eloizo Job \\ Departamento de Física, Química e Biologia,UNESP, Presidente Prudente/SP \\ Paulo de Souza Gonçalves \\ Instituto Agronômico de Campinas/SP
}

Resumo: A borracha natural obtida do látex sangrado de quatro clones de seringueira (Hevea brasiliensis) foi preparada pela coagulação do látex com ácido acético $(10 \%)$ e seca ao ar a $65^{\circ} \mathrm{C}$ (48 horas). Os dados de DMTA foram utilizados para monitorar e avaliar as propriedades termo dinâmico-mecânicas. Os resultados sugerem um possível aumento na massa molecular média do poliisopreno e, também, uma variação em sua distribuição para a borracha natural dos quatro clones de seringueira.

Palavras-chave: Borracha natural, clones, seringueira, propriedades termo dinâmico-mecânicas.

\section{Monitoring and Evaluation of the Raw Natural Rubber Using the Technique of Dynamic Mechanical Thermal Analysis}

Abstract: Natural rubber of latex tapping of four rubber tree clones (Hevea brasiliensis) were prepared by coagulated latex with acetic acid (10\%) and air drying at $65^{\circ} \mathrm{C}$ (48 hours). DMTA data were used to monitor and evaluate the thermo dynamic-mechanical properties. The results suggest a possible increase in the average molecular mass of polyisoprene and also a variation in its distribution of natural rubber for four rubber tree clones.

Keywords: Natural rubber, clones, rubber tree, thermo dynamic-mechanical properties.

\section{Introdução}

A borracha natural é utilizada em uma ampla gama de aplicações industriais, tais como na manufatura de pneus, produtos para uso médico e paramédico, adesivos, calçados e outros. A qualidade de um produto manufaturado de borracha depende da qualidade da borracha natural crua ${ }^{[1]}$.

Nos últimos anos, vêm ocorrendo a busca e a utilização de materiais naturais menos nocivos ao meio ambiente que é beneficiado pela utilização da borracha natural.

O Brasil, que já foi o principal produtor e exportador de borracha no mundo, hoje produz apenas cerca de $1 \%$ da produção mundial, o que é insuficiente para o consumo interno, sendo necessária a importação de aproximadamente $60 \%$ da borracha consumida no país ${ }^{[2]}$. Cabe salientar que, a indústria pneumática é a maior consumidora com consumo de $75 \%$ da produção mundial.

As propriedades físico-químicas da borracha natural variam com o tempo de estocagem, período do ano e origem clona ${ }^{[1,3-5]}$. Além disso, as indústrias estão cada vez mais exigentes em relação à qualidade e uniformidade da borracha natural produzida no país.

A Embrapa Instrumentação Agropecuária, em parceria com o Instituto Agronômico (IAC) tem um programa que avalia clones de seringueira plantados em larga escala e auxilia na recomendação de novos clones no Estado de São Paulo, com o intuito de investigar o efeito das variações de condições climáticas e sazonais na qualidade e uniformidade da BN crua.

O objetivo deste estudo foi utilizar a análise térmica dinâmico-mecânica (DMTA) para monitorar e avaliar o comportamento dinâmico-mecânico da $\mathrm{BN}$ crua de quatro clones de grande difusão no Estado de São Paulo em função do tipo de clone e o período de coleta.

\section{Materiais e Métodos}

As sangrias foram realizadas em doze árvores de cada um dos quatro clones (GT 1, PB 235, IAN 873 e RRIM 600) no município de Votuporanga/SP (Estação Experimental IAC)

Autor para correspondência: Rogério M. B. Moreno, Embrapa Instrumentação Agropecuária, Rua XV de Novembro 1452, Caixa Postal 741, CEP: 13560970, São Carlos, SP. E-mail: rogerio@cnpdia.embrapa.br 
nas datas de: 27/01 (1), 10/02 (2), 24/02 (3), 09/03 (4), 23/03 (5), 06/04 (6), 20/04 (7), 04/05 (8), 18/05 (9), 15/06 (10) e 29/ $06 / 2000$ (11). O látex foi estabilizado com solução de $\mathrm{NH}_{4} \mathrm{OH}$ a $5 \%$, coagulado com solução de ácido acético a $3 \mathrm{~N}$ e o coágulo foi passado em uma calandra para a obtenção de mantas com espessura de $2 \mathrm{~mm}$, sendo seco em estufa à $65^{\circ} \mathrm{C}$.

A plasticidade Wallace $\left(\mathrm{P}_{0}\right)$ foi determinada seguindo a norma ABNT - NBR $11597^{[6]}$.

Na obtenção dos resultados de DMTA foi utilizado o equipamento NETZSCH DMA 242C com interface TASC 414/ $3 \mathrm{~A}$ e controlador 242 . As condições dos ensaios, no modo de tensão de tração, foram: freqüência de $10,0 \mathrm{~Hz}$, faixa de temperatura de -100 a $180{ }^{\circ} \mathrm{C}$, razão de aquecimento: $5^{\circ} \mathrm{C} / \mathrm{min}$ e resfriamento sob atmosfera de $\mathrm{N}_{2}$, sendo a deformação máxima aplicada de 0,025\% com amplitude máxima de 60 $\mu \mathrm{m}$ e uma força dinâmica máxima de $3,0 \mathrm{~N}$ com força estática 1,5X e força dinâmica aplicada. As dimensões dos corpos de prova foram: 10,00 $\mathrm{mm}$ de comprimento, $5,5 \mathrm{~mm}$ de largura e $1,75 \mathrm{~mm}$ de espessura.

\section{Resultados e Discussão}

As Figuras 1 e 2 ilustram a dependência do logaritmo do módulo de armazenamento ( $\log$ E') em função da temperatura.

Dos resultados obtidos (Tabela 1) não se observa um comportamento padrão. Nos casos, onde há um aumento do valor do módulo de armazenamento máximo ( $\mathrm{E}_{\text {máx }}$ ) com o aumento do índice de retenção da plasticidade Wallace $\left(\mathrm{P}_{0}\right)$ pode estar ocorrendo um aumento da massa molecular média (MM)do poliisopreno. A maior MM propicia o maior emaranhamento
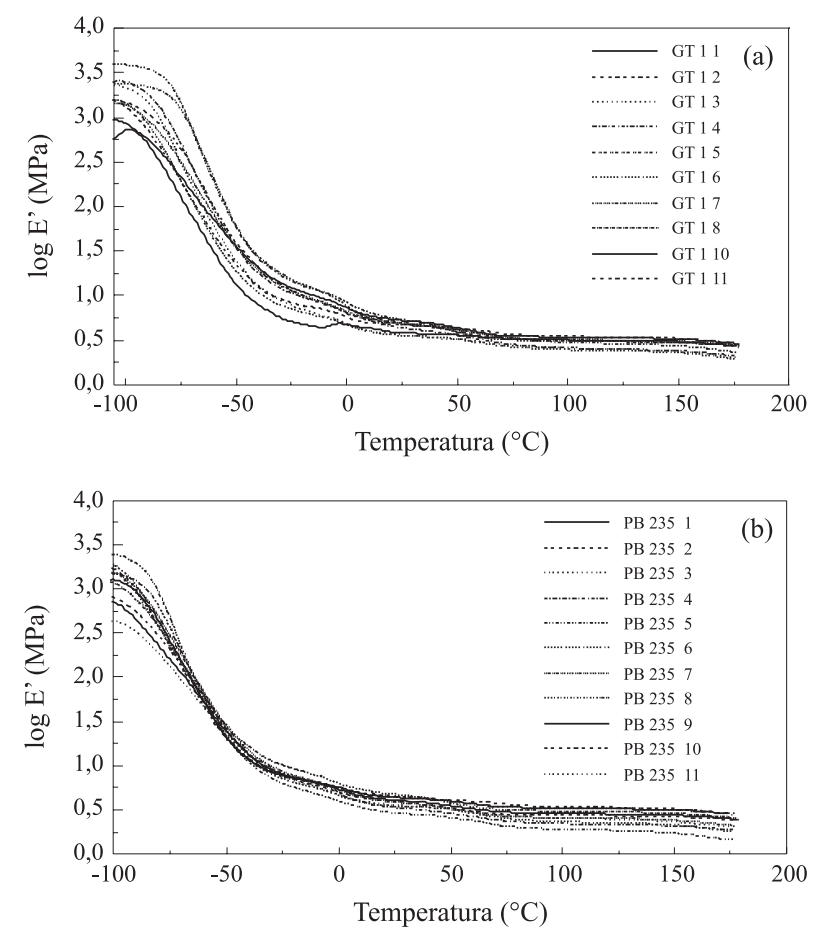

Figura 1. Curvas do logaritmo do módulo de armazenamento[logE' (MPa)] versus temperatura $\left({ }^{\circ} \mathrm{C}\right)$ para as borrachas de onze coletas dos clones GT 1 e PB 235.
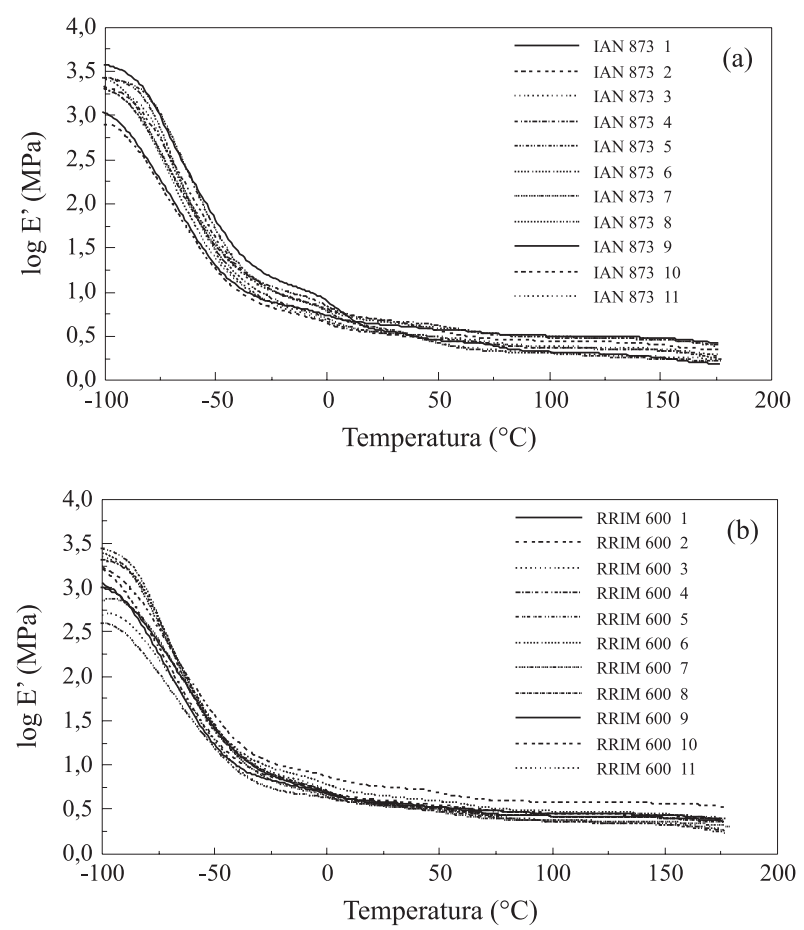

Figura 2. Curvas do logaritmo do módulo de armazenamento[logE' (MPa)] versus temperatura $\left({ }^{\circ} \mathrm{C}\right)$ para as borrachas de onze coletas dos clones IAN 873 e RRIM 600.

das cadeias poliméricas elevando os valores do $\mathrm{E}_{\text {máx }}$, tendo em vista que há uma boa correlação entre $\mathrm{P}_{0} \mathrm{e}$ a massa molar de peso média $\left(\mathrm{M}_{\mathrm{w}}\right)^{[7]}$. Fatores como o percentual de gel, formação de ramificações e a própria distribuição de massa molecular (DMM) podem influenciar nos valores de E'. Os casos, em que o aumento na $\mathrm{P}_{0}$ é acompanhado de um decréscimo no E', podem ser explicados pela possível alteração na DMM. Em geral, a DMM é bimodal e um aumento da fração de menor MM pode funcionar como um plastificante inter$\mathrm{no}^{[8]}$, facilitando o deslizamento das cadeias poliméricas e diminuindo o valor da temperatura de transição vítrea (Tg) e do E' ${ }_{\text {máx }}$ como no caso do clone PB 235 (coleta 9). Nos casos, onde um decréscimo na $\mathrm{P}_{0}$ é acompanhado por um acréscimo do $E_{\text {máx }}$, pode estar ocorrendo um aumento na quantidade de ligações cruzadas (\% de gel) e nas ramificações de cadeia que propiciam um maior impedimento de desenrolamento das cadeias poliméricas favorecendo o aumento do $\mathrm{E}_{\text {máx }}$.

Notam-se variações significativas entre os clones e coletas. As maiores variações no $\mathrm{E}_{\text {máx }}$ foram obtidas pelo clone RRIM 600 e as menores pelo clone IAN 873. Os clones IAN 873 e PB 235 apresentaram o maior e o menor valor médio

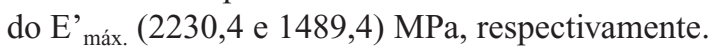

As propriedades da borracha natural crua variam com o tipo de clone e o período de coleta em que a borracha é obti$\mathrm{da}^{[1,3,4]}$. No trabalho realizado por Ferreira et al. $(2002)^{[4]}$ as propriedades da borracha natural crua variaram significativamente em função do tipo de clone, método de sangria e condições climáticas ao longo do ano. O conteúdo de borracha seca no látex (DRC) tende a diminuir no início da estação mais fria e seca (maio a junho), porém, as \% de cinzas e nitrogênio aumentaram no mesmo período. A plasticidade 
Tabela 1. Média $(\overline{\mathrm{X}})$, desvio padrão $(\mathrm{s})$ e o coeficiente de variação $(\mathrm{CV} \%)$ da plasticidade Wallace $\left(\mathrm{P}_{0}\right)$, módulo de armazenamento máximo [E' $\left.{ }_{\text {máx }}(\mathrm{MPa})\right]$ e temperatura de transição vítrea $\left[\mathrm{Tg}\left({ }^{\circ} \mathrm{C}\right)\right]$ das borrachas de onze coletas de látex de quatro clones

\begin{tabular}{|c|c|c|c|c|c|c|c|c|c|c|c|c|}
\hline \multirow[b]{2}{*}{$\mathbf{S}^{*}$} & \multicolumn{3}{|c|}{ GT 1} & \multicolumn{3}{|c|}{ PB 235} & \multicolumn{3}{|c|}{ IAN 873} & \multicolumn{3}{|c|}{ RRIM 600} \\
\hline & $\mathbf{P}_{0}$ & $\begin{array}{l}\mathbf{E}_{\text {max }}^{\prime} \\
\text { (MPA) }\end{array}$ & $\begin{array}{c}\mathbf{T g} \\
\left({ }^{\circ} \mathbf{C}\right)\end{array}$ & $\mathbf{P}_{0}$ & $\begin{array}{l}\mathbf{E}_{\text {max }}^{\prime} \\
\text { (MPA) }\end{array}$ & $\begin{array}{l}\mathbf{T g} \\
\left({ }^{\circ} \mathbf{C}\right)\end{array}$ & $\mathbf{P}_{0}$ & $\begin{array}{l}\mathbf{E}_{\text {max }}^{\prime} \\
\text { (MPA) }\end{array}$ & $\begin{array}{c}\mathbf{T g} \\
\left({ }^{\circ} \mathbf{C}\right)\end{array}$ & $\mathbf{P}_{0}$ & $\begin{array}{l}\mathbf{E}_{\text {max }}^{\prime} \\
(\mathbf{M P A})\end{array}$ & $\begin{array}{c}\mathrm{Tg} \\
\left({ }^{\circ} \mathbf{C}\right)\end{array}$ \\
\hline 1 & 48 & 887,8 & $-77,9$ & 60 & 2446,7 & $-71,0$ & 69 & 1091,8 & $-78,0$ & 79 & 1119,6 & $-73,4$ \\
\hline 2 & 61 & 1348,9 & $-76,0$ & 66 & 1663,6 & $-75,3$ & 67 & 800,1 & $-75,5$ & 79 & 1631,4 & $-74,3$ \\
\hline 3 & 69 & 2149,3 & $-71,5$ & 64 & 1583,4 & $-76,4$ & 73 & 2015,3 & $-70,5$ & 77 & 2236,3 & $-68,4$ \\
\hline 4 & 84 & 2525,6 & $-63,0$ & 70 & 1489,9 & $-67,9$ & 84 & 2043,2 & $-69,8$ & 85 & 2106,0 & $-67,5$ \\
\hline 5 & 66 & 3895,9 & $-69,0$ & 65 & 2446,7 & $-67,5$ & 79 & 2684,8 & $-65,3$ & 72 & 2757,9 & $-68,5$ \\
\hline 6 & 65 & 1466,5 & $-74,5$ & 74 & 1863,6 & $-74,5$ & 76 & 2659,1 & $-74,0$ & 73 & 2487,2 & $-69,4$ \\
\hline 7 & 69 & 1461,2 & $-67,5$ & 79 & 1567,8 & $-69,9$ & 79 & - & $-71,5$ & 84 & 758,4 & $-67,3$ \\
\hline 8 & 59 & 2474,6 & $-58,5$ & 72 & 1311,3 & $-71,0$ & 64 & 2713,3 & $-60,3$ & 71 & 393,8 & $-73,4$ \\
\hline 9 & 78 & - & $-72,8$ & 74 & 726,9 & $-82,5$ & 71 & 3987,5 & $-66,8$ & 75 & 1013,9 & $-68,5$ \\
\hline 10 & 96 & 722,1 & - & 86 & 851,7 & $-66,5$ & 86 & 2196,8 & $-65,4$ & 82 & 1863,4 & $-69,4$ \\
\hline 11 & 81 & 1533,9 & $-63,0$ & 70 & 431,5 & $-74,8$ & 84 & 2112,5 & $-62,3$ & 82 & 293,1 & $-70,3$ \\
\hline $\mathbf{x}$ & 71 & 1846,6 & $-69,4$ & 71 & 1489,4 & $-72,5$ & 76 & 2230,4 & $-69,0$ & 78 & 1514,6 & $-70,0$ \\
\hline $\mathbf{s}$ & 13 & 941,0 & 6,3 & 7 & 644,8 & 4,8 & 7 & 891,3 & 5,6 & 5 & 850,0 & 2,5 \\
\hline cv & 18 & 51,0 & 9,1 & 10 & 43,3 & 6,6 & 9 & 40,0 & 8,1 & 6 & 56,1 & 3,6 \\
\hline
\end{tabular}

*Sangrias
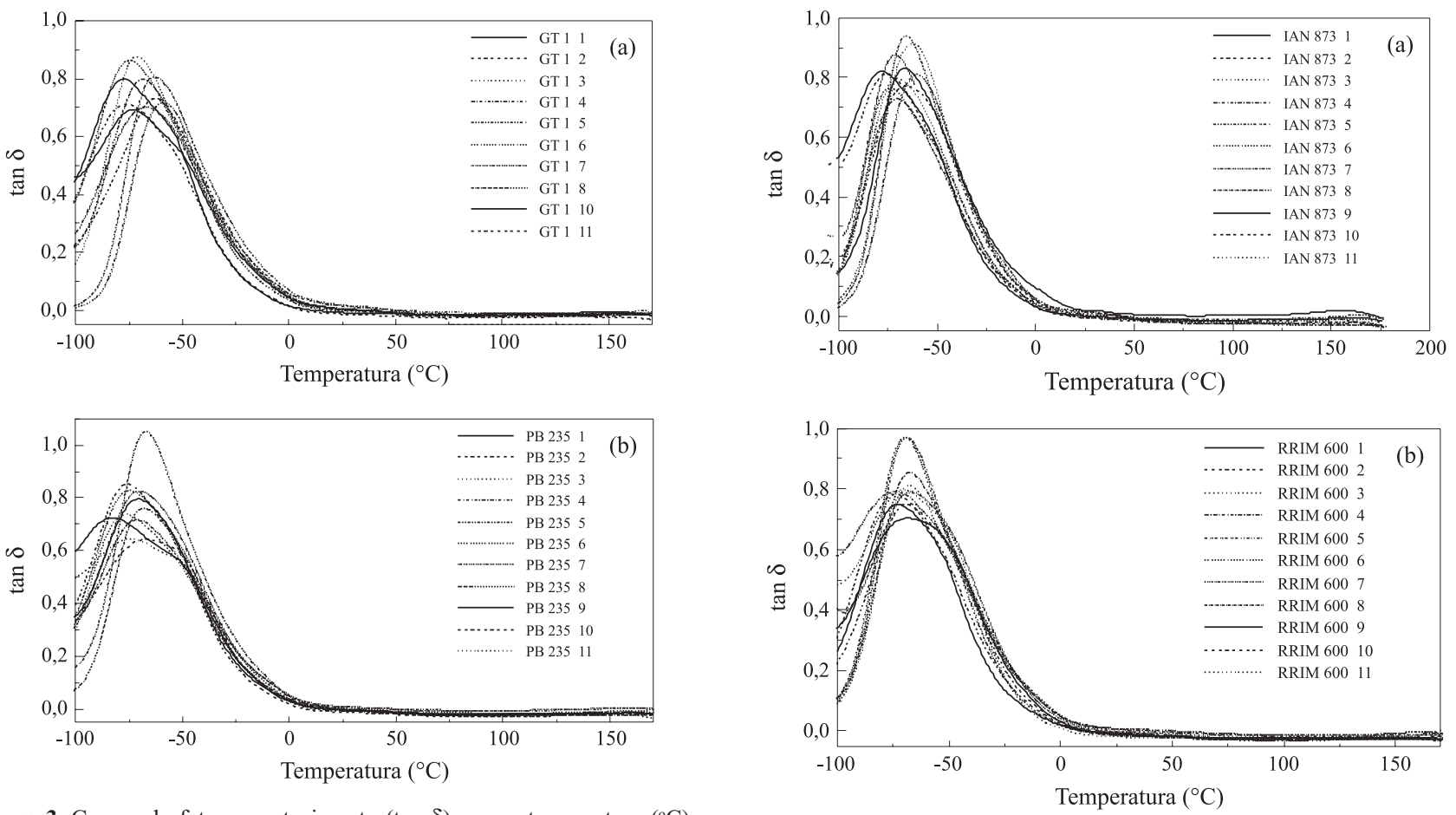

Figura 3. Curvas do fator amortecimento $(\tan \delta)$ versus temperatura $\left({ }^{\circ} \mathrm{C}\right)$ para as borrachas de onze coletas dos clones GT 1 e PB 235

Wallace $\left(\mathrm{P}_{0}\right)$ e a viscosidade Mooney $\left(\mathrm{V}_{\mathrm{R}}\right)$ mostraram uma boa correlação linear e o maior valor médio para essas duas propriedades foi obtido pelo clone RRIM 600.

A faixa de MM é praticamente a mesma para a maior parte dos clones (GT 1, PB 235 e RRIM 600), mas os valores

Figura 4. Curvas do fator amortecimento $(\tan \delta)$ versus temperatura $\left({ }^{\circ} \mathrm{C}\right)$ para as borrachas de onze coletas dos clones IAN 873 e RRIM 600.

médios e os formatos das curvas de DMM são diferentes ${ }^{[8,9]}$. Enquanto clones com borrachas de baixa e média MM apresentam DMM bimodal distinta, clones com borrachas de alta MM usualmente apresentam uma DMM unimodal ${ }^{[9]}$. 
As Figuras 3 e 4 ilustram a dependência do fator amortecimento $(\tan \delta)$ em função da temperatura.

$\mathrm{A} \mathrm{Tg}$ foi medida no ponto máximo da curva do fator amortecimento $(\tan \delta)$ em função da temperatura ${ }^{[10]}$ e apresentou variações distintas nas onze coletas para cada um dos quatro clones. O clone RRIM 600 teve a menor variação na $\mathrm{Tg}$ e o clone GT 1 a maior (Tabela 1). Os materiais poliméricos têm um comportamento viscoelástico $(0,001<\tan \delta<3)^{[11]}$ e os valores obtidos estão dentro dessa faixa.

As variações da MM e na DMM, aliados a variação na quantidade de ligações cruzadas, podem influenciar nos valores $\mathrm{E}_{\text {máx }}$ da borracha natural, o que influenciará nos valores da $\mathrm{Tg}$. Uma maior MM deve, em princípio, diminuir a mobilidade das cadeias poliméricas deslocando as Tg para valores mais altos. De um modo geral, os resultados mostram que um aumento no $\mathrm{E}_{\text {máx }}$ leva a um acréscimo na Tg (Tabela 1).

$\mathrm{Na}$ borracha natural a presença de microgel, macrogel e ramificações na cadeia do poliisopreno têm implicações nas propriedades de interesse tecnológico. Enquanto o macrogel é responsável pelo endurecimento durante a estocagem da borracha natural, a formação do microgel no látex afeta a viscosidade deste que resultará na borracha natural. Diferente do macrogel, que é formado na borracha natural seca sob estocagem, o microgel é formado no látex presente nos vasos laticíferos da seringueira ${ }^{[12]}$. A formação do microgel no látex é iniciada pela condensação de grupos aldeído variando entre 100 e 420 unidades por molécula de poliisopreno ${ }^{[13]}$. Esse processo de condensação também promove o aumento das ligações cruzadas entre as cadeias poliméricas influenciando nos valores da $\mathrm{P}_{0}$ e do $\mathrm{E}_{\text {máx }}$. Como, em geral, a borracha natural possui DMM bimodal, uma maior fração de moléculas com menor $\mathrm{MM}$ pode plastificar a fração de maior $\mathrm{MM}^{[8]}$ aumentando a mobilidade das cadeias poliméricas deslocando a Tg para valores menores.

\section{Conclusões}

Os resultados indicam comportamentos distintos nas propriedades termo dinâmico-mecânicas das borrachas cruas dos quatro clones estudados. Estes comportamentos sugerem a variação, tanto da MM do poliisopreno como da distribuição.

\section{Agradecimentos}

Os autores agradecem o suporte financeiro concedido pela FAPESP, CNPq e Embrapa Instrumentação Agropecuária.

\section{Referências Bibliográficas}

1. Le Roux, Y.; Ehabe, E.; Sainte-Beuve, J.; Nkengafac, J.; Nkeng, J.; Ngolemasango, F.; Gobina, S. - Journal of Rubber Research, 3, p.142, (2000).

2. International Rubber Study Group - Natural Rubber Statistical Bulletin, 58, p.9, (2004).

3. Yip, E. - Journal of Natural Rubber Research, 5, p.52, (1990).

4. Ferreira, M.; Moreno, R. M. B.; Gonçalves, P de S.; Mattoso, L. H. C. - Rubber Chemistry and Technology, 75 , p.1, (2002).

5. Moreno, R. M. B.; Ferreira, M.; Gonçalves, P de S.; Mattoso, L. H. C. - Pesquisa Agropecuária Brasileira, 38, p.583, (2003).

6. Associação Brasileira de Normas Técnicas - Borracha natural: NBR 11597, Rio de Janeiro, 1996, 22 p.

7. Bonfils, F.; Flori, A.; Sainte-Beuve, J. - Journal of Applied Polymer Science, 74, p.3078, (1999).

8. Bonfils, F.; Char, C.; Garnier, Y.; Sanago, A.; Saint-Beuve, J. - Journal of Rubber Research, 3, p.164, (2000).

9. Subramaniam, A. - Rubber Chemistry and Technology, 45, p.346, (1972).

10. Sircar, A. K.; Galaska, M. L.; Rodrigues, S.; Chartoff, R. P. - Rubber Chemistry and Technology, 72, p.513, (1998).

11. Canevarolo, S. V. - Análise dinâmico-mecânica para sistemas poliméricos. Parte 1: $\mathrm{O}$ amortecimento $(\tan \delta)$. Seção tecno-científica, Associação Brasileira de Polímeros, (1991) 36.

12. Sethuraj, M. R. \& Mathew, N. M. - "Natural rubber: Biology, Cultivation and Technology", ELSEVIER, Amsterdam (1992).

13. Burfield, D. R. - Journal of Natural Rubber Research, 1, p.202, (1986).

Enviado: $21 / 11 / 05$

Reenviado: 24/02/06

Aprovado: 06/03/06 